\title{
Der Graf Albert Joseph Hoditz (1706-1778) und das Rosswalder Dominium im Spiegel der Reisebeschreibung von Balthasar Ludwig Tralles "Schattenriss der Annehmlichkeiten von Roswalde"
}

The Count Albert Joseph Hoditz (1706-1778) and the Rosswalder Dominium in the Mirror of the Travel Description by Balthasar Ludwig Tralles "Schattenriss der Annehmlichkeiten von Roswalde"

Iveta Zlá

\begin{abstract}
The article deals with the picture of Count Albert Joseph Hoditz and his castle in Rosswald in the above-mentioned travel description of Balthasar Ludwig Tralles. The contribution is initiated by an insight into the biographical information about Tralles. The Count Albert Joseph Hoditz is presented at the time of the cultural flourishing of his castle. The travel description accentuates his cultural commitment, which deserves the attention of European literary and cultural history.
\end{abstract}

\section{Keywords}

Travel description, stay in Rosswald, cultural flowering, the time between Baroque and Enlightenment, German-language literary history, European cultural history 


\section{Einführung in die Thematik}

Das Rosswalder Schloss und sein mit der künstlerischen Vielfalt, Extravaganz und Gastfreundlichkeit des Grafen Albert Joseph Hoditz verbundenes Renommee lockte in dieses Dominium viele Künstler, führende Persönlichkeiten des zeitgenössischen gesellschaftlich-politischen Lebens und nicht zuletzt Reisende. Das Bild dieser Schlossresidenz ist in die Reisebeschreibung von Balthasar Ludwig Tralles eingegangen, die in lateinischer ${ }^{1}$ und deutscher Sprache ${ }^{2}$ verfasst wurde. Der Reisebericht thematisierte die von Tralles wahrscheinlich 1756 unternommene Reise nach Rosswald und seinen Aufenthalt in dieser Schlossresidenz.

Obwohl die Reisebeschreibung eine ganze Reihe von Informationen über die künstlerische Atmosphäre am Rosswalder Hof vermittelt, schwankt sie zwischen wissenschaftlichem und dem literarischem Reisebericht. Die Überschneidung der fiktiven und dokumentarischen Ebene ist für diese Gattung kennzeichnend, was auch die folgenden Worte Wolfgang Strucks verdeutlichen: „Reiseberichte erzählen nicht nur von Grenzüberschreitungen; sie vollziehen selbst Grenzgänge. [...] Grenzgänge zwischen Erfahrung und Sinn, Poesie und Wissen, Fiktion und Dokumentation, Text und Welt, zwischen der rhetorischen Strategie der ,evidentia', des Vor-Augen-Stellens einer ,als-ob'-Wirklichkeit, und der philosophischen und naturwissenschaftlichen Evidenz als Grundlage moderner Wissensproduktion."3

Im Vorbericht der Reisebeschreibung bietet Balthasar Ludwig Tralles einen Einblick in seine Beziehung zum Grafen Hoditz, den er hochgeschätzt hat und der ihn um Folgendes gebeten hat: „Sein [des Grafen Albert Joseph Hoditz ] zu starkes Vertrauen in meine geringen Kräfte reizte ihn gnädig von mir zu fordern, daß ich eine Beschreibung vieler [...] wunderswerther und merkwürdiger Sachen [von seinem Rosswalder Aufenthalt] in einem deutschen Gedicht liefern möchte. "4 Obwohl sich Balthasar Ludwig Tralles der Reisebeschreibung zugewendet hat, hat er dieses Verlangen des Grafen erfüllt und diesen Adligen sowie die künstlerische Atmosphäre seines Dominiums dargestellt. Im Unterschied zur wissenschaftlich ausgerichteten Reisebeschreibung, der die Vermittlung des enzyklopädisch gezeichneten Faktenwissens und der Verlust des narrativen Elements eigen sind, ${ }^{5}$ sind die Erinnerungen Tralles' an seinen Rosswalder Besuch durch eine bunte Palette der stilistischen Darstellungsmittel und „einige alte dichterische und mythologische Blümchen"6 gekennzeichnet. Dementsprechend tauchen bereits im einführenden Kapitel dieser Reisebeschreibung die folgenden Worte auf, die die literari-

1 Vgl. TRALLES, Balthasar Ludwig: Amoenitatum Roswaldensium adumbratio. Breslau 1773.

2 Vgl. TRALLES, Balthasar Ludwig: Schattenriss der Annehmlichkeiten von Roswalde. Breslau 1776.

3 Zitiert nach: https://www.uni-erfurt.de/uni/dienstleistung/presse/pressemitteilungen/2008/68-08/ zuletzt geöfftnet am 20. 10. 2013.

4 TRALLES, Balthasar Ludwig: Schattenriss der Annehmlichkeiten von Roswalde. Breslau 1776, S. 3-4.

5 Vgl. http://www.jungeforschung.de/reise/genre\%20handout.pdf/zuletzt geöffnet am 15. 7. 2014.

6 TRALLES, Balthasar Ludwig: Schattenriss der Annehmlichkeiten von Roswalde. Breslau 1776, S. 5. 
schen Ambitionen ihres Autors andeuten: „Wir wollen auf einige Zeit Breslau verlassen; meine Muse wird uns begleiten."

Die vorliegende Studie setzt sich zum Ziel, das Bild des Grafen Hoditz, sein kulturelles Engagement und das künstlerische Panorama des Rosswalder Hofs in der Reisebeschreibung von Balthasar Ludwig Tralles darzulegen und ihre poetischen Facetten zu erfassen. Darüber hinaus konzentriert sich die Abhandlung im literaturwissenschaftlichen Fokus auf die Auseinandersetzung mit der im Reisebericht umgesetzten Strategie der „evidentia“ sowie mit ihrer Funktion und ihren Intentionen, die mit der Darstellung des Grafen Hoditz und seines Herrschaftsguts zusammenhängen. Dennoch werden die literarischhistorischen Berührungspunkte der Reisebeschreibung nur zum Teil, vorwiegend in den entsprechenden Fußnoten angedeutet. Die aufgezeichneten Parallelen der literarischen Gestaltung mit dem realen Hintergrund werden lediglich als eines der Ausgangspunkte für die literarische Analyse betrachtet.

\section{Balthasar Ludwig Tralles als deutschsprachiger Autor und Literaturkritiker. Ein Abriss}

Obwohl Balthasar Ludwig Tralles (1708-1797) vor allem durch seine medizinischen Erforschungen $^{8}$ und philosophisch-theologischen Schriften ${ }^{9}$ Berühmtheit erlangte, lässt sich von der Tätigkeit dieses Gelehrten sein literarisches Schaffen nicht trennen. Die wissenschaftlichen Schriften sowie das literarische Werk dieses Gelehrten sind in der Universitätsbibliothek Wrocław überliefert und einige Handschriften von Ludwig Balthasar Tralles ${ }^{10}$ sind in der Bibliothek Albertina Leipzig ${ }^{11}$ befindlich.

Ins Jahr 1768 fällt der Aufenthalt von Tralles in Rosswald, der in seine Reisebeschreibung „Schattenriss der Annehmlichkeiten von Roswalde“12 eingegangen ist. Der Reisebericht ist durch eine ganze Reihe von literarischen Merkmalen gekennzeichnet und balanciert zwischen der wissenschaftlichen und künstlerischen Reisebeschreibung.

\section{Ebenda, S. 6.}

8 Vgl. TRALLES, Balthasar Ludwig: Exercitatione physico-medica virtutem Camphorae refrigerantem. Breslau 1734; ders: Historia cholerae atrocissimae, quam sustinuit ipse, persanavit aegerrime, atque in usus publicos adjectis animadversionibus theoretico-practicis. Breslau 1753; ders: Usus Opii Salubris Et Noxius In Morborum Medela: Solidis Et Certis Principiis Superstructus. 4 Bde. Breslau 1757-1762; ders: Usus vesicantium salubris et noxius in morborum medela. 2 Bde. Breslau 1782; ders: De vena jugulari frequentius secanda. Breslau - Leipzig 1735 etc.

9 Vgl. TRALLES, Balthasar Ludwig: De Machina Et Anima Humana Prorsus. Breslau - Lepizig 1749; ders.: Schrift- und Vernunft-mäßige Gedancken von der Gegenwart Christi bey dem H. Abendmahl, ehedessen in der Sprache der Gelehrten diesen allein gewidmet, nunmehro aber zur Erhaltung seines guten Nahmens allen vernünftigen Christen vor die Augen geleget. Breslau 1755 etc.

10 Die Handschriften schließen die Zeitspanne 1759-1764 ein.

11 Vgl. DÖRING, Detlef: Katalog der Handschriften der Universitäts-Bibliothek Leipzig. Bd. 1, Teil 4. Wiesbaden 2005, MS 01308.

12 TRALLES, Balthasar Ludwig: Schattenriss der Annehmlichkeiten von Roswalde. Breslau 1776. 
Die literarischen Reflexionen von Balthasar Ludwig Tralles sind mit der deutschen Literaturkritik verbunden. Er schätzte in seinen literaturkritischen Abhandlungen die von Johann Christoph Gottsched proklamierten poetischen Grundsätze hoch und stellte sich polemisch gegen die dramatische, vom englischen elisabethanischen Theater ausgehende Konzeption Gotthold Ephraim Lessings.

1781 ist die Schrift Friedrich II. „De la Littérature Allemande“13 erschienen, in der der preußische Monarch die zeitgenössische deutsche Literatur kritisch betrachtet hat. Dieses Werk war stark auf die Hochschätzung der französischen Literatur orientiert und der König betrachtete die deutschsprachige Literatur der Aufklärung und den mit ihr zusammenhängenden gesellschaftlich-kulturellen Wandel des Bürgertums unter dem Aspekt des französischen literarischen Bildes in der zweiten Hälfte des 18. Jh. Die Schrift Friedrichs II. hat negative Reaktionen verzeichnet. Balthasar Ludwig Tralles stellte sich gegen die Herabsetzung der deutschen Literatur, die Friedrich II. in „De la Littérature Allemande“ zum Ausdruck gebracht hat. Die Abhandlung von Tralles „Schreiben von der deutschen Sprache und Literatur bey Gelegenheit der zu Berlin im Jahre 1781 in französischer Sprache herausgekommenen vortrefflichen Schrift: Über die deutsche Literatur; über die Mängel“14 ist nicht nur als Apologie der deutschen literarischen Tradition, sondern auch als „ein beachtenswerther Beitrag zur Geschichte des literarischen Urteils in Deutschland"15 zu verstehen.

Das literarische Schaffen und die literaturkritischen Beiträge von Balthasar Ludwig Tralles haben in der deutschsprachigen Literaturgeschichte des 18. Jh. sowohl positiven als auch negativen Nachhall hinterlassen. Obwohl sein literarisches Werk für fehlende poetische Kraft kritisiert wurde, zeichnet es sich durch epigrammatische Tiefe aus und stützt sich auf einige mit den literarisch-gesellschaftlichen Kontakten von Tralles verbundene biographische Momente.

\section{Das literarische Bild des Grafen Albert Joseph Hoditz und des Rosswalder Herrschaftsguts in der Reisebeschreibung von Balthasar Ludwig Tralles „Schattenriss der Annehmlichkeiten von Roswalde"}

Das kulturelle Engagement des Grafen Albert Joseph Hoditz, die die künstlerischen und literarischen Affinitäten evozierende Atmosphäre seines Dominiums und nicht zuletzt die Gastfreundlichkeit dieses Adligen haben einen Anziehungspunkt für zahlreiche Besucher dargestellt. Die Reisebeschreibung von Balthasar Ludwig Tralles geht von der Faktualität seines in den 70er Jahren des 18. Jh. verwirklichten Aufenthaltes in Rosswad

13 Vgl. FRIEDRICH II.: De la Littérature Allemande. Stuttgart 1883.

14 Vgl. TRALLES, Balthasar Ludwig: Schreiben von der deutschen Sprache und Literatur bey Gelegenheit der zu Berlin im Jahre 1781 in französischer Sprache herausgekommenen vortrefflichen Schrift: Über die deutsche Literatur; über die Mängel. Breslau 1782.

15 http://www.deutsche-biographie.de/sfz82866.html/ zuletzt geöffnet am 23. 7. 2013 
aus und ist durch die Analepse gekennzeichnet, was die folgenden Worte belegen: „[Ich] fuhr fort, die süssen Bilder lebhaft in mein Gedächtnis zurückrufen [...]." ${ }^{16}$ Die Literarisierung der Erlebnisse und Impressionen vom Rosswalder Besuch zeichnen sich durch elliptische Auslassung einiger Begebenheiten aus: „Ich gestehe gerne, daß ich vieles übrig gelassen, was ich gar nicht berührt [habe]." ${ }^{17}$ Die erzählte Zeit schließt vier Tage ein und von der Wiedergabe der Ereignisse lässt sich nicht die Zeitraffung trennen.

Der Erzähler macht primär auf den Ruf der Rosswalder Schlossresidenz aufmerksam, die er metaphorisch als "glücklichen Winkel der Erde"18 bezeichnet. Das literarische Bild des Rosswalder Schlosses wird im Reisebericht von Tralles durch die Personifikation sowie durch die als Hendiadyoin arrangierte Verbindung von Adverbien „getreu und untrüglich“19 erfasst, durch die die suggestive Aufwertung des Rosswalder Dominiums auffällt: „[...] der verbreitete Ruf des Ortes wird uns durch seine Stimme locken und getreu und untrüglich führen. “20

In diesem Kontext wird der Graf Hoditz hochgeschätzt und der Adlige wird als „Schöpfer, Regierer und Meister“21 bezeichnet. Der angeführten Textpassage ist nicht zuletzt eine Charakteristik des Aussehens von Hoditz zu entnehmen. Das Erscheinungsbild des Grafen Hoditz wurde durch eine ganze Reihe von positiv geprägten Metaphern gekennzeichnet, deren Kumulation den panegyrischen Unterton der Textstelle in den Vordergrund rückt: „[...] seine offene Stirne ist heiter; aus den glänzenden Augen leuchtet bewunderswerthe Güte; sanfte Freundlichkeit herrscht auf seinen Lippen und alle Gesichtszüge verrathen die grosse Seele, die sich nur in Wohlthaten gefällt."22

Darüber hinaus wird das literarische Bild des Grafen Hoditz sowie der Rosswalder Landschaft in der Reisebeschreibung von Tralles als eine Symbiose der Natur und Kunst aufgefasst. Der Autor setzt teils ein poetisches Mosaik von Asyndeta um, teils betont er die Verknüpfung der Natur mit Kunst durch die klimaktische Steigerung, um ihre Phänomenalität zu akzentuieren. Dem Erzähler legt er dabei Folgendes in den Mund: „Aber der kluge und sinnreiche Greis vermag Sie auch zu gewinnen, zu reizen, zu treiben, zu locken, zu nötigen, zu zwingen, [...] um Sie dem Dienst der Kunst zu überlassen"23

Die Reisebeschreibung ist nicht zuletzt durch die Hervorhebung der Annäherung an die Natur geprägt, die der Lebensweise von Hoditz eigen war. ${ }^{24}$ Die Sehnsucht

16 TRALLES, Balthasar Ludwig: Schattenriss der Annehmlichkeiten von Roswalde. Breslau 1776, S. 5.

17 Ebenda.

18 Ebenda, S. 7 f.

19 Ebenda, S. 7.

20 Ebenda.

21 Vgl. ebenda, S. 8.

22 Ebenda, S. 8 f.

23 Ebenda, S. 11.

24 Die Lebensweise des Grafen Hoditz wurde von seiner Neigung zur Natur gekennzeichnet, was sich u. A. in der das Schloss Rosswald umgebenden Gartenlandschaft zeigte. Die im Reisebericht thematisierte Einstellung dieses Adligen zum Leben an metropoliten Höfen belegen auch historische Erforschungen dieser Problematik. Vgl. STILLER, Max: Chronik der Markt Rosswald, S. 20 f. In: ZAO, FVH, Inventarnr. 163, Kartonnr. 93; MYŠKA, Milan: a. a. O., S. 100 f.; Bein, Werner: „Der mährische Epikuräer“ Albert Joseph Hoditz (1706-1778) 
des Grafen nach der ruhigen und inspirativen Atmosphäre des Landeslebens sowie seine Distanz zur Welt der großstädtischen Höfe werden durch einige Exkurse über bedeutende antike Persönlichkeiten und mythologische Figuren ins positive Licht gerückt. Die Exkurse über die Zuwendung der ländlichen Lebensweise von Quintius, Cato etc. unterstreichen im literaturwissenschaftlichen Fokus die Präsenz des auktorialen Point of View, durch die der Erzähler im dargelegten Reisebericht gekennzeichnet ist. Der Vergleich der genannten Denker und mythologischer Gestalten mit den biographischen Facetten über den Grafen Hoditz zielt gleichsam auf die Hochachtung dieses Adligen ab. Darüber hinaus ist die Rolle der Exkurse, die die lineare Narration der Reisebeschreibung brechen, durch den literaturhistorischen Background zu begründen. Das literarische Schaffen von Tralles wurde durch die barocke Literatur beeinflusst und gleichsam schloss es prägende Impulse der Aufklärung ein. Die Einbettung dieser Informationen in das erste Kapitel des Reiseberichts ist als Zeichen der barocken Gelehrsamkeit zu verstehen.

In Anknüpfung an diese Textpassage wird wiederholt die ländliche Lebensweise des Grafen Hoditz mit biographischen Informationen über einige berühmte Persönlichkeiten verglichen, um die Vorteile der Lebenswelt dieses Adligen zu betonen. Obwohl Albert Joseph Hoditz sowohl in der historisch ausgerichteten Forschungsliteratur ${ }^{25}$ als auch in der europäischen Literatur ${ }^{26}$ als zwischen Genialität und Narrheit stehende Persönlichkeit charakterisiert wurde, wird der Graf Hoditz in der Reisebeschreibung von Tralles als „der unvergleichliche Weltweise“27 bezeichnet.

Das Lob des Landlebens wird nicht zuletzt in den Überschriften der aufeinander folgenden Kapitel gesteigert, indem die „Anmut des Landlebens“28 zur „Pracht des Landlebens zu Roswalde“ ${ }^{29}$ avanciert. Dem vielfältigen Kolorit des Rosswalder Hofs wird das Geschehen in Tarent, Tibur und Laurentiana gegenübergestellt, die mit den Aufenthalten von Cicero, Horaz und Plinius verbunden sind. Die Lobpreisung des Rosswalder Dominiums wird durch die Akkumulation von Adjektiven ${ }^{30}$ intensiviert, die im Kontext der angeführten antiken Topoi die Einzigartigkeit und Wichtigkeit der Rosswalder Residenz verdeutlichen.

In den Kontext der antiken Zitate von Euripides, Lucan, Ovidius, Horatius etc. wird die Schilderung des Mittagessens mit dem Grafen Hoditz eingebettet, die durch zahlreiche Metaphern ${ }^{31}$ durchdrungen ist. Darüber hinaus wird die Nachzeichnung der kulina-

als Mäzen der Schönen Künste. In: SCHLAGER, Karlheinz (Hg.): Festschrift Hubert Unverricht. Tutzing 1992, S. 38.

25 Vgl. WOLNY, Gregor: Taschenbuch für Geschichte Mährens und Schlesiens. Bd. 2. Brünn 1826, S. 206. Zitiert nach: BEIN, Werner: „Der mährische Epikuräer“ Albert Joseph Hoditz (1706-1778) als Mäzen der Schönen Künste. In: SCHLAGER, Karlheinz (Hg.): Festschrift Hubert Unverricht. Tutzing 1992, S. 37.

26 Vgl. z. B. RICHTER, Johannes: Der Wundergraf. Ein närrisches Genie - ein genialer Graf. Berlin 1937.

27 TRALLES, Balthasar Ludwig: Schattenriss der Annehmlichkeiten von Roswalde. Breslau 1776, S. 14.

28 Ebenda, S. 11.

29 Ebenda, S. 15.

30 Vgl. Ebenda, S. 16.

31 „Es bleibe dem Pythagoras das gerechte Lob, daß er unter einem brennenden Himmelstrich, seinem 
rischen Genüsse durch Akkumulationen ${ }^{32}$ und Diäresen ${ }^{33}$ thematisiert und die Lebensweise des Grafen Hoditz skizziert.

Vom Rosswalder kulinarischen Mosaik und Essgewohnheiten in dieser Schlossresidenz ließ sich die durch literaturwissenschaftliche Darstellungsmittel geprägte Auseinandersetzung mit dem musikalischen Leben am Rosswalder Hof nicht trennen. Der auktoriale Erzähler weist auf das kulturelle Engagement des Grafen Albert Joseph Hoditz mit den folgenden Worten hin: „Diese Gewohnheit der Vorfahren [Musik zu pflegen], beobachtet der Erlauchte Graf, ein ächter Kenner und Beförderer aller schönen Künste, auf das beste. " ${ }^{34}$ In der Textpassage wird auf die musikalische Kontinuität aufmerksam gemacht, die der Graf Hoditz weiterentwickelt. Obwohl der Erzähler das musikalische Kolorit des Rosswalder Dominiums in den Vordergrund rückt, bezeichnet er den Grafen als „Kenner und Beförderer aller schönen Künste“ ${ }^{35}$ womit er den mit Musik verknüpften thematischen Rahmen übersteigt. Die Hochschätzung dieses Adligen wird durch die superlativische Form des Adverbs "gut" gesteigert, die die Einzigartigkeit der kulturellen Aktivitäten von Hoditz auffallen lässt. Die Skizzierung des Musiklebens in der Rosswalder Schlossresidenz wird von Vergleichen mit den mythologischen Sagen ${ }^{36}$ durchdrungen, deren Schwerpunkt das Motiv des Musikalischen darstellt.

In der Reisebeschreibung von Tralles wurde die Zuwendung des Grafen Hoditz zu den tragenden Gedanken der Aufklärung verewigt, unter denen die Ausbildung, Belehrung und Erziehung dominieren. Der analysierte Text zeichnet sich durch einen Aufbau aus, der zur Hervorhebung der Wichtigkeit von Ausbildung und Anerkennung der erzieherischen Verdienste des Grafen Hoditz beiträgt. Die Textpassage ist durch einige Fragen eingeleitet, die Aufmerksamkeit und Neugier der Leser wecken: „Woher ein so gelehriges und fruchtbares Genie? Woher die brennende Nacheiferung? Woher das Bestreben nach Tugend und Rechtschaffenheit, und woher die löbliche Begierde nach Ruhm und Ehre?" ${ }^{\text {"37 }}$ Diese Fragen beantwortet der auktoriale Erzähler, indem er die Tatsache unterstreicht, dass in Rosswald lediglich die Adeligen leben müssen. Die angeführte These wird jedoch geleugnet, um die Phänomenalität der didaktischen Bemühungen von Hoditz zu akzentuieren, dessen Bestrebungen auf arme Menschen ausgerichtet sind. Die Betonung dieser Verdienste erfolgt durch ein Exclamatio, an das ein allegorisch gezeichnetes Bild der menschlichen Erziehung und Bildung anknüpft. In diesem Kontext gewinnt der Graf Hoditz die Attribute eines hochausgebildeten und im künstlerischen Bereich bewanderten

Volk, [...] den sparsamen Genuß des Fleisches empfohlen. [...] Sey wahr, daß die ersten [...] daß sie von Speisen, die aus dem Schoos der Erde hervorsprießen, gelebt haben." TRALLES, Balthasar Ludwig: Schattenriss der Annehmlichkeiten von Roswalde. Breslau 1776, S. 26.

32 Es ist aber hier in reichlicher Menge Oesterreichischer, Rheinwein, Burgunder, Champager [...], Ungarischer [...]." Ebenda, S. 41.

33 „[...] wir kommen zu der kalten Waare [...] des Naschwerks Confect, Kuchen, Plätzchen, Zwieback, Tortchen, eingemachten Früchten, Marzipan [...]." Ebenda, S. 37.

34 Ebenda, S. 48.

35 Ebenda.

36 In diesem Zusammenhang werden die Sagen über Orpheus und Euridike und über Amphion sowie der Arion-Mythos thematisiert. Vgl. ebenda, S. 48 f.

37 Ebenda, S. 61. 
Adeligen, der die Ausbildung verbreitet. Die Ausbildung wird mit Musen und Grazien metaphorisch in Verbindung gebracht und die Wichtigkeit der erzieherischen Bemühungen des Grafen sowie das Ausbildungsniveau der Rosswalder Ausbildungsanstalten werden durch Epitheta betont. Die an der untersuchten Textstelle ${ }^{38}$ auftauchenden Allegorien akzentuieren die Rolle der Ausbildung in der Rosswalder Schlossresidenz.

Durch zahlreiche Kommentare des Erzählers, denen der auktoriale Point of wiev eigen ist, wird der Einblick in die Atmosphäre der Rosswalder Schlossresidenz gekennzeichnet. Der Akzent liegt dabei neben der Schilderung der Essgewohnheiten auf der Skizzierung der Gesprächsthemen, die für die Gäste dieses Dominiums als zentral galten. Die Darlegung der angeführten Thematik balanciert zwischen den neutral gehaltenen Betrachtungen bis zu den panegyrischen Darstellungen der Atmosphäre im Rosswalder Schloss. Die Hochschätzung des Grafen Hoditz und seiner Lebenswelt geht argumentativ von der Kritik der Essgewohnheiten und Nahrung im Allgemeinen aus, um auf die Vorzüge der Rosswalder Lebensweise hinzuweisen. In den Gesprächen der Gäste von Rosswald dominiert eine thematische Vielfalt, die sich in Anhäufung der zu Asyndeta stilisierten Substantive demonstriert: „Angenehm ist es, mit Leuten, die an Geburt, Glücksgütern, Wissenschaft, Würde, Lebensart [...] verschieden sind, [...] in Gesellschaft zu seyn, ihre Vertraulichkeit, Freundschaft, Gunst und Gnade sich zu gewinnen und Gesprächen so mancherley Inhalts zuzuhören. "39 Die Gespräche der Rosswalder Gäste variieren von Jura über Geschichte, Philosophie und Politik bis zur Literatur. Ihr literarisches Bild oszilliert im Reisebericht von Tralles um die rhetorischen Prinzipien der Evidentia und ist wertender Eingriffe des Erzählers frei.

Einen Einblick in das kulturelle Geschehen des Rosswalder Schlosses bietet das kennzeichnend betitelte Kapitel „Die Oper“, das von Pluralis auctoris eingeleitet ist und den Leser in die Handlung einbezieht. Die Analyse dieser Textstelle erfasst einen positiven Nachhall der Opernaufführung. Obwohl der Reisebericht nicht einen Einblick in das Repertoire des Rosswalder Schlosstheaters bietet, weist der Erzähler auf das Vorspiel zur Opernaufführung durch Folgendes hin: „Auf den Schauplatz tritt von ungezähmter Liebesflamme durchaus glühend Pancraz, der mit marktschreierischer Geschwätzigkeit und Burlesken-Anstand das marmorne Herz seiner angebeteten Isabel zu beugen und zu weichen sucht." ${ }^{40}$ Die Schilderung der Handlung dieses Vorspiels verdeutlicht ihre den Hanswurst-Aufführungen ähnlichen Züge, die in der zweiten Hälfte des 18. Jh. der Kritik ausgesetzt waren. Dennoch wurden die schauspielerischen Leistungen vom auktorialen Erzähler mit Lob und Anerkennung charakterisiert und das Kapitel mit dem Zitat aus „Aeneid“ Vergils abgeschlossen, durch das die Hochachtung des Rosswalder künstlerischen Milieus akzentuiert wurde.

Die Darstellung der Atmosphäre im Rosswalder Schloss wird durch zahlreiche Exkurse durchdrungen, denen eine thematische, zwischen den neutralen und kritischen

38 Auf die Wichtigkeit der Ausbildung bezieht sich ebenfalls die folgende Allegorie: „Wer nicht zu gehöriger Zeit ackert und einsäet, wird sich keiner reichlichen Aerndte zu erfreuen haben; und wer nicht in den ersten Tagen des Lenzens die Blütchen pflegt, wird wenig oder gar keine Früchte erhalten." Ebenda, S. 64.

39 TRALLES, Balthasar Ludwig: Schattenriss der Annehmlichkeiten von Roswalde. Breslau 1776, S. 74.

40 Ebenda. 
Einstellungen variierende Vielfalt eigen ist. Sie stellt einen Hintergrund dar, vor dem die Phänomenalität des Rosswalder Dominiums auffällt. Die Hervorhebung der Gastfreundlichkeit und des intellektuellen Esprits von Hoditz wird nicht zuletzt durch seinen Vergleich mit Platon, Cato und Cicero erzielt und durch die adjektivischen Superlativformen ${ }^{41}$ gesteigert.

Obwohl die Reisebeschreibung „Schattenriss der Annehmlichkeiten von Roswalde“ in der historisch ausgerichteten Forschungsliteratur ${ }^{42}$ als durchaus panegyrisch charakterisiert wird, wurden im Reisebericht die Rosswalder dramatischen Aufführungen kritisiert. Der auktoriale Erzähler moniert die Regellosigkeit und Unnatürlichkeit der Schauspiele, was diese Worte belegen: „Mir scheint diese Gattung von Schauspielen viel Regelloses und Unnatürliches an sich zu haben, ob sie schon gleichsam mit fortdauernder Zauberey durch die schnelle Abwechslung und Verwandlung der Gegenstände ausserordentlich lebhaft wirkt [...] ${ }^{\text {“43 }}$ Diese Textstelle korrespondiert mit den Informationen über die Rosswalder Theaterszene im Zeithorizont 1755 -1761, durch deren Einfluss die Aufführungen im Rosswalder Schlosstheater auch in folgenden Dezennien gekennzeichnet werden durften. Die kritisch gemeinten Reflexionen weisen auf die Rolle des Phantastischen im Rosswalder Theaterrepertoire hin, das die zentrale Rolle auf dieser Theaterbühne spielte und sich nicht mehr an die von der Poetik Johann Christoph Gottscheds ausgehenden Prinzipien hielt. Den in der Reisebeschreibung thematisierten Überlegungen wird in dieser Textpassage explizit Authentizität verliehen: „Der erlauchte Graf willigt darein, indem wir aufstehen und erlaubt mir, nichts zu verbergen, sondern die verstecktesten Meinungen meines Herzens [über die Rosswalder Theateraufführungen] frei vorzulegen und zu entwickeln." ${ }^{44}$

Das literarische Schaffen des Grafen Hoditz wird in der Reisebeschreibung von Tralles jedoch hingegen positiv gewertet und mit dem dichterischen Werk Albrecht Hallers verglichen: „Er [der Graf Albert Joseph Hoditz] liest mir [...] seine erhabenen und kraftvollen Gedichte verschiedenen Inhalts vor, die ich weil sie nach Hallers Ader schmecken, voll Bewunderung beglückwünschend anhöre. "45 Die skizzierten literarischen Facetten der Dichtung des Grafen Hoditz verraten ihre Kommensurabilität mit den prägenden Tendenzen der Aufklärung, deren lehrhafte Intentionen sowohl im literarischen Schaffen von Hoditz als auch von Tralles Niederschlag gefunden haben.

Mit den historisch relevanten Informationen korrespondiert das Bild der Rosswalder Bibliothek ${ }^{46}$ und der hiesigen Gartenlandschaft. In der Reisebeschreibung wird die thematische Vielfalt des Bücherinventars hervorgehoben, das geistes- sowie naturwissenschaftlich ausgerichtete Publikationen beinhaltet. Die Darstellung der Bibliothek von Hoditz in diesem Reisebericht zeichnet sich sowohl durch ihre sachliche Beschreibung und affirmative Erzählhaltung aus, als auch ist sie durch die semantische Aufwertung

41 Vgl. ebenda, S. 86.

42 Vgl. z. B. MYŠKA, Milan: a. a. O., S. 307; Bein, Werner: a. a. O., S. 36.

43 TRALLES, Balthasar Ludwig: Schattenriss der Annehmlichkeiten von Roswalde. Breslau 1776, S. 87.

44 Ebenda.

45 Ebenda, S. 98.

46 Vgl. MYŠKA, Milan: a. a. O. S. 190-208. 
gekennzeichnet. Darüber hinaus ist sie von zahlreichen Akkumulationen durchdrungen, durch die ihr inhaltlicher Umfang akzentuiert wird. Die Bedeutsamkeit der Rosswalder Büchersammlung wird nicht zuletzt durch die anaphorischen Wiederholungen ${ }^{47}$ hervorgehoben, die die thematische Vielfalt sowie das Ausmaß der Rosswalder Bibliothek unterstreichen. Darüber hinaus wird der thematische Umfang des Bibliotheksinventars nicht nur durch ein Exclamatio betont, sondern auch die superlativischen Adjektivformen tragen zur Hervorhebung seiner Einzigartigkeit bei: „Welch ein Vorrath! Da sind Weltsysteme, Globen und Instrumente, die zur Mathematik, Physik, Optik, Astronomie und Mechanik gehören. Da sind Gemälde von den grössten Meistern, gestickte Teppiche, zahlreiche Bildnisse der trefflichsten Künstler in Kupfer gestochen, die artigsten Produkte der Färbekunst [...]." ${ }^{* 48}$

Mit den historisch relevanten Ausführungen korrespondiert das in der Reisebeschreibung von Tralles thematisierte Bild des Rosswalder Schlossgartens, ${ }^{49}$ das auf die Hervorhebung seines vielfältigen Gepräges ausgerichtet ist. Diese Reichhaltigkeit der Rosswalder Gartenszenerie demonstriert sich in den literarischen und historischen Affinitäten und in der Atmosphäre, die durch die Kreativität des Grafen Hoditz geprägt ist. In der Fülle von Motiven dominieren Darstellungen, die der griechischen und orientalischen Mythologie entnommen wurden. Das Bild des Rosswalder Gartens in der Reisebeschreibung von Tralles wird durch einige Exclamatia gekennzeichnet, die nicht nur die Aufmerksamkeit des Lesepublikums wecken, sondern auch die Pracht des Rosswalder Gartens in den Vordergrund rücken. Das Augenmerk wird dominant auf die bildhauerischen Werke gerichtet, die das natürliche Gepräge der Gartenlandschaft mit artifiziellen Merkmalen verbinden. Die die Figuren der griechischen Mythologie und orientalische Motive verkörpernden Stauen hinterlassen den Eindruck der Authentizität, was die folgenden Worte des auktorialen Erzählers wiedergeben: „Hier steht ein blitzschwingender Jupiter von Stein, blutlos und unbeseelt, aber kühn da, als ob er lebendig wäre; dort eine marmorne Venus, als ob sie spräche, mit lebendigen Zügen in ihrem Antlitz." ${ }^{50}$ Im Reisebericht von Tralles werden jedoch auch christliche Motive thematisiert, die im Schlossgarten Niederschlag gefunden haben. Neben der christlichen Symbolik verdeutlicht der Erzähler Hintergründe der mythologischen Geschichten, in denen die im Rosswalder Garten abgebildeten Figuren von Dianes, Arethusa, Neptun sowie von Najaden, Nereiden, Sirenen etc. eine zentrale Rolle spielen. Die Einbettung der ausgewählten Gestalten in das thematische Gewebe der antiken Mythologie unterstreicht wiederum die Dominanz der auktorialen Erzählsituation und die Phänomenalität des Rosswalder Dominiums und lassen den Einblick des Erzählers in die antike Literatur auffallen. Er appelliert nicht nur an die Aufmerksamkeit und Neugier der Leser, sondern sie werden auch durch Pluralis auctoris in die Handlung einbezogen.

47 „Welch ein Vorrath! Da sind Weltsysteme, Globen und Instrumente, die zur Mathematik, Physik, Optik, Astronomie und Mechanik gehören. Da sind Gemälde von den grössten Meistern, gestickte Teppiche, zahlreiche Bildnisse der trefflichsten Künstler in Kupfer gestochen, die artigsten Produkte der Färbekunst [...].“ TRALLES, Balthasar Ludwig: Schattenriss der Annehmlichkeiten von Roswalde. Breslau 1776, S. 102.

48 Ebenda.

49 Vgl. ZAO, FVH, Inventarnr. 163, Kartonnr. 93: Stiller, Max: Chronik der Markt Rosswald, S. 20 f.

50 Ebenda, S. 107. 
Einige der in der Reisebeschreibung von Tralles erfassten antiken Figuren schmücken Springbrunnen, Wasserkanäle sowie -fälle, auf deren Anzahl die rhetorischen Fragen hinweisen: „Wer aber wohl könnte alle diese immerfliessenden Wasseradern, deren es über vier tausend gibt, erzählen? Wer alle die prächtigen Gestalten der Springbrunnen, welche sie durch erstaunliche Kunst gewonnen, beschreiben? Wer schilderte so viele unnachahmliche Wasserfälle, deren seufzende, geschwätzige, murmelnde Wellen sich aus der Höhe [...] herabstürzen?" ${ }^{\text {"51 }}$ Der Erzähler weist explizit auf die Einzigartigkeit der im Rosswalder Garten befindlichen Wasserwerke hin und suggeriert ausmalend die Atmosphäre dieses Ortes durch personifizierte Adjektivattribute.

Der Kommentar des auktorialen Erzählers lässt sich vom Plot des als „Der Tanz“ betitelten Kapitels nicht trennen. Die Schilderung des Rosswalder musikalischen, durch den Tanz gekennzeichneten Bildes bildet einen Vorspann für den Einblick in ausgewählte historisch-kulturelle Meilensteine der europäischen Tanzentwicklung mit dem Ziel, das mit dem Rosswalder Dominium verknüpfte kulturelle Milieu hervorzuheben. Darüber hinaus wird der Tanz im Kontext der Auseinandersetzung mit Reflexionen der antiken Denker wie Platon, Seneca, Sokrates etc. thematisiert, die sowohl die belehrenden Intentionen der Reisebeschreibung verdeutlichen, als auch auf die repräsentative Rolle des Tanzes sowie der Musik im Rosswalder Schloss hinweisen.

Im Reisebericht von Tralles wird die Handlungslinie einer unbetitelten Komödie nachgezeichnet, die der Erzähler als „ein sehr artiges und dem Geist unserer Zeit angemeßenes Schauspiel“ ${ }^{“ 52}$ charakterisiert. Vor ihrem Hintergrund wird die Nachahmung der Wirklichkeit als eines der prägenden Momente des Dramas in den Vordergrund gerückt, wodurch auch die künstlerischen Überlegungen von Tralles artikuliert werden. Dies deutet auch das in die Reisebeschreibung eingegliederte und als „Urteile über die Schauspiele“ betitelte Kapitel an, das sich durch die Dominanz des auktorialen Point of View auszeichnet und die durch die Thematisierung des Grafen Albert Joseph Hoditz und seines Herrschaftsguts geprägte Handlungslinie bricht. Die im Reisebericht vorgestellten Ausführungen über das Drama gehen von der aristotelischen Poetik aus, akzentuieren die Nachahmung der Natur sowie die erzieherischen Intentionen und den unterhaltenden Unterton des Dramas als seine grundlegenden Charakteristika.

Die Schilderungen der Rosswalder Gartenlandschaft bilden einen Hintergrund, vor dem die Einzigartigkeit dieses Dominiums sowie die Gastfreundschaft und Ausbildung des Grafen Hoditz hochgeachtet werden. Dieser Adlige wird nicht nur im Spiegel seiner epikureischen Lebensweise ${ }^{53}$ nachgezeichnet, sondern er wird auch als ein alter, mit schlechtem Gesundheitszustand kämpfender Graf charakterisiert. Dennoch wurde Hoditz im Reisebericht von Tralles folgendermaßen dargestellt: „So lange er sich unter diesen noch befindet,

\footnotetext{
51 Ebenda, S. 108.

52 Ebenda, S. 126.

53 Die Lebensweise des Grafen Albert Joseph Hoditz wurde von Friedrich II. und in der einschlägigen Forschungsliteratur als epikureisch bezeichnet. Vgl. z. B.: Bein, Werner: a. a. O., S. 35-45; MYŠKA, Milan: a. a. O., S. 316 etc.
} 
vergißt er großmüthig seines Kummers und trägt die zu empfindlichen Anfälle mit der Ruhe eines Weltweisen, und beinahe stoischen Standhaftigkeit und Stärke des Geistes “54

Durch Pluralis auctoris wird die Schilderung des Schäferfestes eingeleitet, das im Rosswalder Schlossgarten veranstaltet wird. Der Leser wird in die Handlung dieses Kapitels nicht nur durch den Autorenplural eingezogen, sondern das metaphernreiche Bild der Gartenlandschaft steigert auch seine Aufmerksamkeit und weist auf die Phänomenalität dieses Ortes hin: „, [...] betreten wir die Wiese, hier und da mit murmelnden Bächen bewässert, die im lebhaftesten Grün glänzt, und mit Kräutern und wohlriechenden Blumen besäet ist, deren ambrosischen Hauch die sanften Winde ringsum verbreiten und uns wohltätig zuwehen. ${ }^{\text {"55 }}$

In die Reisebeschreibung sind einige von Tralles vermittelte Gedichte eingegangen, die während des Schäferfestes vorgetragen wurden und mit ausgewählten Figuren der antiken Mythologie verbunden waren. Unter diesen Figuren dominierte der Gott Arkadiens Pan, der sowohl in der antiken Mythologie als auch in diesen Schäferspielen die Rolle des Beschützers der Hirten übernommen hat. Für Pales wurde das folgende Gedicht vorgetragen, in dem die Nymphen und Najaden thematisiert wurden:

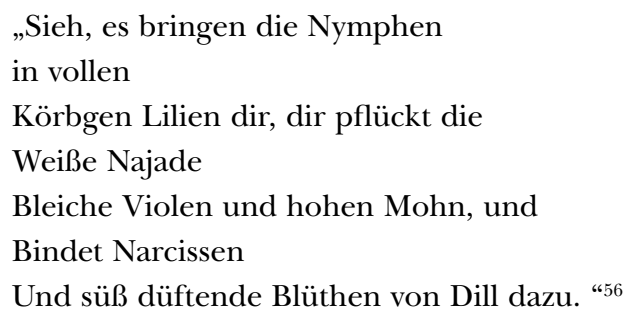

Die Verbindung der bukolisch geprägten, reimlosen Dichtung mit der Rosswalder Gartenszenerie rückt die Natur in den Vordergrund und räsoniert mit einer der tragenden Ideen der Aufklärung, die die Annäherung an die Natur durchsetzt. Die im Gedicht erfassten Nymphen und Najaden sind als Verkörperung der Naturkräfte und Begleiterinnen hoher Gottheiten ${ }^{57}$ zu verstehen. Die Natur wird nicht nur durch die im Gedicht variierten Blumen thematisiert, sondern die Blumen assoziieren auch die Farben, die sich von der Natur nicht trennen lassen. Durch die als Anadiplose stilisierte Wiederholung des Personalpronomens „dir“ werden die Begrüßung der Göttin Pales Athena und ihre Beschenkung mit Blumen intensiviert.

Die Schäferdichtung wird in der Reisebeschreibung von Tralles inhaltlich umrissen. In den Reisebericht werden die Auszüge aus diesem unbetitelten Schäfergedicht eingegliedert. Die lobenden Facetten dieser Textstelle werden durch Folgendes verdeutlicht: „Es ist dieses Gedicht seines Urhebers Apolls würdig, der nicht von dem Himmel ver-

54 TRALLES, Balthasar Ludwig: Schattenriss der Annehmlichkeiten von Roswalde. Breslau 1776, S. 163.

55 Ebenda.

56 Ebenda, S. 165.

57 TRALLES, Balthasar Ludwig: a. a. O., S. 165. 
wiesen, sondern vom Himmel herab den Roswalder Fluren vergönnt geworden zu seyn scheint, um da selbst [...] die schönen Künste zu lehren, und Musik und Dichterkunst auf eine solche Höhe zu bringen. ${ }^{\text {"58 }}$ Das Rosswalder kulturelle Milieu wird metaphorisch mit dem antiken Gott der schönen Künste, Musik und Dichterkunst verknüpft, um auf das hohe künstlerische Niveau dieses Dominiums hinzuweisen. Da die Schäferdichtung im Rosswalder Garten vorgetragen wird, wendet sich der Erzähler der Skizzierung dieser Gartenlandschaft zu. Er weist implizit auf die Verbindung der Natur mit der Welt der Literatur und Musik hin, die jedoch in das Reich der Bildhauerkunst und Gartenarchitektur eingebettet werden. Durch diese Intentionen ist auch der Abschlussteil der Reisebeschreibung geprägt, in dem der Beschreibung des Druidentempels Aufmerksamkeit geschenkt wird. Im Reisebericht wird dieses Bauwerk von Druidenstatuen umgeben und in ihren Umkreis gesetzt.

Durch Bewunderung ist die Darstellung der Feuer- und Wasserspiele gekennzeichnet, durch die das Schäferfest beendet wurde. Der auktoriale Erzähler reflektiert seine Einzelbeobachtungen, stellt sie in einem Gesamtbild vor und rundet sie mit den folgenden Worten ab: „Mit welchem Vergnügen verstreicht uns dieser bis in die späte Nacht verlängerte Tag, dessen Gedächtnis keine Vergessenheit auslöschen soll! Mit welchem herrlichen Ende wird das Hodizische Werk gekrönt!"599 Die im Reisebericht von Tralles verewigten Impressionen werden als Exclamatia stilisiert und akzentuieren das Lob dieses Festes.

Einen Kontrapunkt zur Schilderung der dem Rosswalder Dominium eigenen künstlerischen Vielfalt, der abwechslungsreichen Ausprägungen des Schlossgartens sowie des Naturells und der Lebensweise des Grafen Hoditz stellt die in den Abschlusskapiteln thematisierte Erinnerung der verstorbenen Ehefrau des Grafen Hoditz dar: „O, wie schnell war das Schauspiel dieses kostbaren Lebens beendigt! Ach! Es fiel mit bleyerner Schwere der dichte und schwarze Vorhang, den niemand mehr aufheben kann." ${ }^{\text {"60 }}$ Der zitierten Textstelle sind die emotionale Regungen evozierenden sprachlichen und rhetorischen Mittel wie Interjektionen und Exclamatia zu entnehmen, durch die die Aufmerksamkeit des Lesers gesteigert wird. Das Lebensende wird metaphorisch als der für immer gefallene Vorhang dargestellt, der gleichsam die Zäsur zwischen Leben und Tod sowie das mit Angst wahrgenommene Unbekannte andeutet. Darüber hinaus korrespondiert die Thematisierung der Todesthematik mit der symbolischen Bedeutung der schwarzen Farbe, die für Tod und Trauer kennzeichnend ist. Das Andenken der Gräfin Wilhelmine Friederike Sophie von Sachsen-Weißenfels ist nicht nur mit der Nachzeichnung der in der Rosswalder Gartenlandschaft errichteten Trauerallee verknüpft, sondern es regt auch die vom auktorialen Erzähler artikulierten Reflexionen über die Eitelkeit des menschlichen Lebens an. Eine symbolische Bedeutung dieser Textpassage hängt nicht zuletzt mit ihrer Stellung in der Reisebeschreibung zusammen, die die zwei letzten Kapitel einschließt und gleichsam als Abschied vom Grafen Hoditz aufgefasst ist.

58 Ebenda, S. 166.

59 Ebenda, S. 186.

60 Ebenda, S. 189. 


\section{Schlussfolgerungen}

Die Reisebeschreibung „Schattenriss der Annehmlichkeiten von Roswalde“ weist sowohl literarische als auch dokumentarische, historisch ausgerichtete Schwerpunkte auf. Sie ist temporal strukturiert und nach den von Tralles in Rosswald verbrachten Tagen eingeteilt. Dennoch vermisst diese Gliederung die konkreten Zeitangaben, die die mit dem Rosswalder Aufenthalt verknüpfte Zeitspanne markieren würden. Diese historischen Informationen sind ebenfalls nicht aus dem Vorbericht der Reisebeschreibung abzuleiten. Die in Rosswald verbrachten Tage werden retrospektiv thematisiert, wodurch der Erzählstandort geprägt ist. Diese zeitliche Entfernung schwächt die Authentizität der Narration ab, die von direkter Rede frei ist und sich durch Zeitraffung auszeichnet.

Obwohl der Rosswalder Besuch retrospektiv thematisiert wird, ist seine Schilderung dominant durch Präsens gekennzeichnet. Diese Tempusverwendung sowie die zeitlichthematische Strukturierung des Reiseberichts dürften die Intention involvieren, der Reisebeschreibung Plastizität und Glaubwürdigkeit zu verleihen. Die zeitliche Distanz wird in den Kommentaren des auktorialen Erzählers verdeutlicht, die im Präteritum verfasst wurden und unter dem Aspekt der Modalität ihnen der Indikativ eigen ist. Dies korrespondiert mit den thematischen Akzenten der Ausführungen, die vorwiegend auf die Reflexionen der antiken Denker eingehen und auf einen fundierten Einblick des Erzählers in diese Problematik hinweisen.

Die Erzählhaltung schwankt zwischen der affirmativen und euphemistischen Prägung, trotzdem ist der Reisebericht durch eine panegyrische Dominanz gekennzeichnet. Das Lob des Grafen Hoditz und des Rosswalder Schlosses wird durch einige Strategien erzielt. Die Hervorhebung der positiven Charaktereigenschaften des Grafen, seiner Ausbildung, Kreativität und seiner künstlerischen Begabung wird an einigen Textstellen explizit zum Ausdruck gebracht. Das kulturelle Engagement dieses Adligen, seine Bemühungen um die Durchsetzung der Ausbildung sowie die Einzigartigkeit seines Dominiums fallen durch die Vergleiche mit berühmten antiken Persönlichkeiten und Kulturstätten des Altertums auf. Ihre Aufwertung erfolgt nicht zuletzt durch die Eingliederung der Informationen über den Grafen Hoditz und die Rosswalder Schlossresidenz in die Kommentare des auktorialen Erzählers, in deren Kontext dieses Dominium an der Peripherie den kulturellen Metropolen gleichgestellt wird. Die panegyrischen Züge der Reisebeschreibung werden durch die Umsetzung von zahlreichen metaphernreichen Ausführungen, Personifikationen, Hyperbeln, adjektivischen Akkumulationen, Superlativformen etc. geprägt. Die panegyrischen Facetten des Reiseberichts involvieren ebenfalls die fiktionalen Merkmale, die in ihrer Neigung zur Übertreibung und stellenweise zum einseitigen Lob bestehen. Trotzdem sind der Reisebeschreibung auch kritische Bemerkungen des auktorialen Erzählers zu entnehmen, die auf die künstlerische Konzeption der Rosswalder Theateraufführungen eingehen.

Obwohl der Graf Hoditz durchaus hochgeachtet wird, wird er ebenfalls als ein Greis mit Vor- und Nachteilen des fortgeschrittenen Alters vorgestellt. Dementsprechend wurde dieser Adlige in der Reisebeschreibung von Tralles als keine typisierte bzw. idealisierte Figur dargelegt. Dies ist neben der Invention des Autors durch die Schwan- 
kung des Reiseberichts zwischen der dokumentarischen und literarischen Ebene zu begründen.

Die Überschneidung der historisch ausgerichteten und literarischen Linie der Reisebeschreibung lässt sich von der rhetorischen Figur der „evidentia“ nicht trennen. Die Darstellung des Rosswalder Aufenthaltes geht von den Impressionen eines historischen Zeugen aus, der in die Reisebeschreibung seine subjektiven, nicht selten emotiv geprägten Erlebnisse projiziert. Durch die Verbindung des ekphrastischen Prinzips der Evidenz mit dessen panegyrisch-fiktiver Schattierung sowie mit der Schwankung zwischen der historischen Realität und dem literarischen Bild wird die historische Relevanz des erforschten Reiseberichts abgeschwächt. Dennoch wurden im Reisebericht - neben seinen literarischen Facetten - historische Informationen vermittelt, deren detaillierte Untersuchung jedoch unter dem kulturhistorischen Aspekt zu erforschen ist.

\section{Quellen und Literatur}

BEIN, Werner (1992): „Der mährische Epikuräer“ Albert Joseph Hoditz (1706-1778) als Mäzen der Schönen Künste. In: SCHLAGER, Karlheinz (Hg.): Festschrift Hubert Unverricht. Tutzing, S. 35-45.

DÖRING, Detlef (2005): Katalog der Handschriften der Universitäts-Bibliothek Leipzig. Bd. 1, Teil 4. Wiesbaden, MS 01308.

FRIEDRICH II (1883).: De la Littérature Allemande. Stuttgart.

MYŠKA, Milan: Hrabě Hodic a jeho svět. Zámecká kultura ve Slezsku mezi barokem a osvícenstvím. Ostrava 2011.

TRALLES, Balthasar Ludwig (1734): Exercitatione physico-medica virtutem Camphorae refrigerantem. Breslau.

TRALLES, Balthasar Ludwig (1735): De vena jugulari frequentius secanda. Breslau - Leipzig.

TRALLES, Balthasar Ludwig (1753): Historia cholerae atrocissimae, quam sustinuit ipse, persanavit aegerrime, atque in usus publicos adjectis animadversionibus theoretico-practicis. Breslau.

TRALLES, Balthasar Ludwig (1757-1762): Usus Opii Salubris Et Noxius In Morborum Medela: Solidis Et Certis Principiis Superstructus. 4 Bde. Breslau.

TRALLES, Balthasar Ludwig (1776): Schattenriss der Annehmlichkeiten von Roswalde. Breslau.

TRALLES, Balthasar Ludwig (1782): Schreiben von der deutschen Sprache und Literatur bey Gelegenheit der zu Berlin im Jahre 1781 in französischer Sprache herausgekommenen vortrefflichen Schrift: Über die deutsche Literatur; über die Mängel. Breslau.

TRALLES, Balthasar Ludwig (1782): Usus vesicantium salubris et noxius in morborum medela. 2 Bde. Breslau.

ZAO (Zemský archiv v Opavě/Landesarchiv Troppau), FVH (Fond Velké Hoštice/Fond Großhoschütz), Inventarnr. 163, Kartonnr. 93. 


\section{Internetlinks}

https://www.uni-erfurt.de/uni/dienstleistung/presse/pressemitteilungen/2008/68-08/zuletzt geöfftnet am 20. 10. 2013.

http://www.jungeforschung.de/reise/genre\%20handout.pdf/zuletzt geöffnet am 15. 7. 2014.

http://www.deutsche-biographie.de/sfz82866.html/zuletzt geöffnet am 23. 7. 2013

doc. Mgr. Iveta Zlá, Ph.D. / iveta.zla@osu.cz

Ostravská univerzita, Filozofická fakulta, Katedra germanistiky,

Reální 5, 70103 Ostrava, CZ 